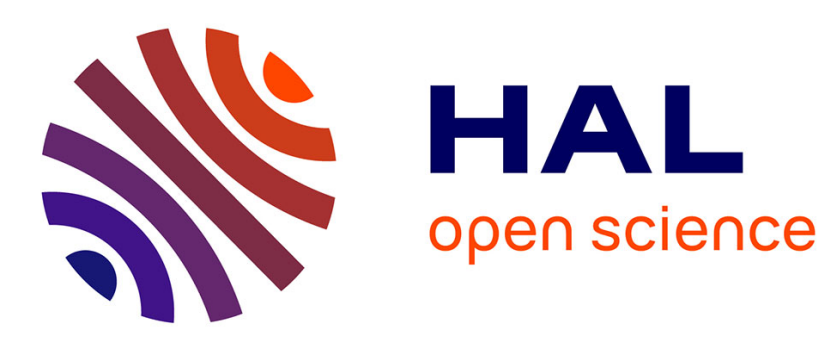

\title{
Geometry optimization of helical swimming at low Reynolds number
}

\author{
Johan E Quispe, Ali Oulmas, Stéphane Régnier
}

\section{To cite this version:}

Johan E Quispe, Ali Oulmas, Stéphane Régnier. Geometry optimization of helical swimming at low Reynolds number. 2019 International Conference on Manipulation, Automation and Robotics at Small Scales (MARSS), Jul 2019, Helsinki, Finland. pp.1-6, 10.1109/MARSS.2019.8860937 . hal-03309704

\section{HAL Id: hal-03309704 https://hal.science/hal-03309704}

Submitted on 30 Jul 2021

HAL is a multi-disciplinary open access archive for the deposit and dissemination of scientific research documents, whether they are published or not. The documents may come from teaching and research institutions in France or abroad, or from public or private research centers.
L'archive ouverte pluridisciplinaire HAL, est destinée au dépôt et à la diffusion de documents scientifiques de niveau recherche, publiés ou non, émanant des établissements d'enseignement et de recherche français ou étrangers, des laboratoires publics ou privés. 


\title{
Geometry optimization of helical swimming at low Reynolds number
}

\author{
Johan E. Quispe, Ali Oulmas and Stéphane Régnier \\ Institut des Systèmes Intelligents et Robotique (ISIR) \\ Sorbonne Université \\ Paris, France \\ quispe@isir.upmc.fr \\ oulmas@isir.upmc.fr \\ stephane.regnier@isir.upmc.fr
}

\begin{abstract}
This paper aims to improve magnetic helical swimming varying the cross-section geometry and considering a conical geometry for helical robot. Both parameters have not been extensively studied in the literature. The conical helix is generated when the helix radial pitch varies constantly and due to that, it could be used in delivery tasks of big-sized millimetric objects. To conceive the whole study, experimentation, and finite-element-method (FEM) simulations are performed in order to corroborate the experimental data in the literature. The influence on the propulsion of the helix cross-section geometry in a cylindrical helix, and the number of turns in a conical helix was demonstrated. Results show that helices with triangular cross-sections provide better propulsion than circular ones, these latter widely used in various micro- and nanoswimmer designs in literature. This result could change the way that helical swimmers are manufactured today. Besides, conical helices with 1-turn and 1.5-turn have shown better performances than multi-turn ones in terms of speed and propulsion force. From this part of the study, the main contribution has been the novel design proposed for delivery tasks.
\end{abstract}

Index Terms-helical swimmers, optimization, low Reynolds number.

\section{INTRODUCTION}

Over the years, helical swimmers have been extensively studied because of their capabilities to move in hard-toreach and viscous environments that could serve in several future applications such as non-invasive medicine [6], [10], micro- and nanomanipulation [4], [13], and among others. These swimmers mimic the typical corkscrew movement performed by some prokaryote cells such as Escherichia coli or Rhodobacter sphaeroides bacteria [9]. Nowadays, a good understanding of the helix dynamic in stationary fluids has been achieved thanks to the great amount of literature on this topic [5], [7], [12]. Nonetheless, there are still some gaps to fill in order to finally utilize this robot in in vivo applications such as in targeted drug delivery through the human body. For instance, until now the flow behavior within the human body has not been so well modeled due to the complex dynamic generated by the non-homogeneous geometry of the conduits. Moreover, the mixture of different Newtonian and non-Newtonian fluids that compose our bodily fluids, and

This work was supported by the French National Research Agency through ANR MultiFlag (grant number ANR-16-CE33-0019). the change of the flow regime because of unforeseen events (heart attacks, respiratory failures, anxiety, etc) that can not be circumvented [11], have not permitted to date modeling the flow behavior under these difficult conditions. Furthermore, strategies to insert and/or extract the robot and the choice of the robot composition in order to achieve the required biocompatibility and/or biodegradability [17] has not been fully mastered. Other issues concern the design of a system to effectively actuate the robot along its 3D path and an ideal medical imaging system to get accurately the robot coordinates in real time [8]. Despite all these previous issues, researchers have put a lot of efforts to clear up step by step each issue presented with the final goal of developing these envisioned technologies.

During the last years, researchers have studied how to improve the swimming performances varying different geometric parameters of the helix. Among these attempts, [16] demonstrated by design of experiments (D.O.E.) that the parameter which influences more on the propulsion speed is the pitch value. The larger the pitch, the larger the propulsion speed. Other several works have found better performances for oneturn helix than the multi-turn ones [1], [3], [14]. Moreover, a simulation work [2] based on past studies have developed one strategy to optimize the helical shape by maximizing the mobility matrix coefficients using a boundary integral equation. Among the parameters studied, the cross-section orientation with respect to the helix tangent, and the pitch value have shown a great influence on the propulsion speed, and so on. Furthermore, it is known that an important nongeometric parameter, which influences on the performance, is the magnetization. [15] has confirmed while having a strong magnetization the magnetic force will be larger and will be able to overcome the robot inertia in order to rotate it for a long range of frequencies, in other words, the larger the magnetization the larger the step-out (or cut-off) frequency. Notwithstanding the above considerations, it seems that researchers have not studied extensively the influence of the cross-sectional area on the helix propulsion.

This manuscript gives a clear explanation of helical swimmer dynamics, and new parameters which influence as well on the propulsion speed but that have not been well studied. Among 


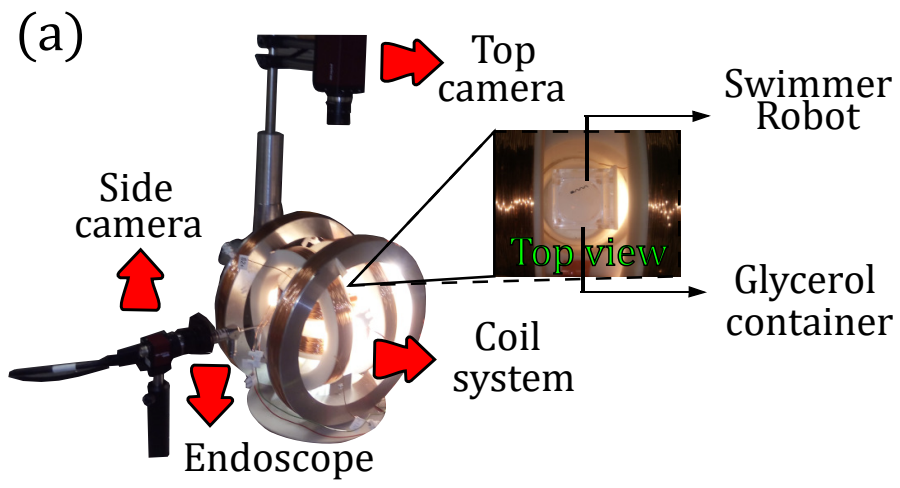

(b)

Fig. 1. Technical details. (a) Experimental setup composed basically of 3 pairs of Helmholtz coils, two cameras, an endoscope and a LED array around the glycerol container. (b) The rotating magnetic field (blue) generated by the coil system allows rotating synchronously the magnet magnetization (green) providing a propulsion force practically perpendicular to the rotating magnetic field plane thanks to the fluid-structure interaction.

these parameters in this study, the cross-section geometry in a cylindrical helix and the number of turns in a conical helix were taken into account.

To sum up, the first study consists of analyzing the impact of the cross-section geometry on the propulsion speed while the second is focused on the impact of the number turns on propulsion speed of a conical helix with a fixed radial pitch. The former study shows that the triangular cross-section improves the propulsion speed before reaching the cut-off or step-out frequency $(4.5 \mathrm{~Hz}$ in our case). The latter study demonstrates that 1.0-turn and 1.5-turn helices improve propulsion speed. These studies were corroborated by FEM simulation results which agree with the experimental data, contributing to state of the art for the design of helical swimmers.

Currently, it is a well-known fact that actuation by low-strength magnetic means does not damage the human body since it does not strongly alter the chemical composition of our cells. In that sense, magnetic actuation seems to be the solution for the helical robot, and for that reason, the experimental part of this work has been performed using a nested Helmholtz coil system, the technical details of this set-up and the experiments are given in section II. A deeper explanation of the theoretical background, and the experimental and simulation results are given in Sections III and IV, respectively. Finally, conclusions and the contribution of this work are stated in section V.

\section{EXPERIMENTAL SETUP AND TECHNICAL DETAILS}

The experimental setup is composed of 3 pairs of Helmholtz coils arranged perpendicularly and capable of $10 \mathrm{mT}$ as maximum. Each pair of coils is driven by an ADS 50/5 4-Q-DC servoamplifiers of Maxon motor, capable of 5A continuous current and 10A peak current. These servoamplifiers are alimented by a TDK-Lambda power supply, capable of $6.7 \mathrm{~A}$ current and $48 \mathrm{~V}$ as output tension. The analog communication between the PC and the amplifiers is accomplished by a Sensory 626 Analog and Digital I/O card. Finally, to track the position of the robot, two cameras and an endoscope are used for the top and side views (cf. Fig1.a). The system is capable of generating a uniform rotating magnetic field in the workspace (space bounded by the smaller coils) in order to propel the millimeter robot (cf. Fig1.b). The swimmer robots are conceived using VisiJet M3 black material and manufactured by a 3D printer machine. The magnetization for all different designs is given by a Neodymium magnet disk with $0.5 \mathrm{~mm}$ in thickness and $1.5 \mathrm{~mm}$ in diameter inside of a cylindrical head with $2.5 \mathrm{~mm}$ in height and $0.8 \mathrm{~mm}$ in radius (cf. Fig1.b). Finally, in order to reproduce a low Reynolds number environment, these robots are immersed in glycerol at $23^{\circ} \mathrm{C}$ on average and viscosity 1.08Pa.s.

\section{THEORETICAL BACKGROUND}

The helical microswimmer locomotion has been well studied in several works simplifying dramatically the dynamic model [5], [9]. In those works, the non-fluidic force $\mathbf{F}$ and torque $\boldsymbol{\tau}$ are related to the velocity $\mathbf{V}$ and rotation frequency $\boldsymbol{\omega}$ in a linear fashion by the mobility matrix. That relation is defined as follows [5]:

$$
\left[\begin{array}{l}
\mathbf{F} \\
\boldsymbol{\tau}
\end{array}\right]=\left(\begin{array}{cc}
A & B \\
B^{T} & C
\end{array}\right)\left[\begin{array}{l}
\mathbf{V} \\
\boldsymbol{\omega}
\end{array}\right]
$$

The previous equation could be defined in a 2 d.o.f. model with all its components being scalars. This simplification provides a good approximation of helical behavior as described by Purcell [9]. However, in order to have a complete understanding of how the helical swimmer behaves in 3 dimensions, [5] provided a 6 d.o.f model using the resistive force theory (RFT). In that case $A, B$ and $C$ are $3 \times 3$ matrices which depend on the geometry of the swimmer and the fluid viscosity while $\mathbf{F}, \boldsymbol{\tau}, \mathbf{V}$ and $\boldsymbol{\omega}$ are $3 \times 1$ vectors. The approach of RFT is simply to determine the forces caused by the velocity $\left(\mathbf{v}_{s}\right)$ of the infinitesimally small segments of the helix when rotating. Each contribution produced by each length is decomposed into components which are parallel or perpendicular to the segment and then relates with their parallel $d \mathbf{f}_{\| s}$ and perpendicular $d \mathbf{f}_{\perp s}$ generated drag forces.

$$
\begin{aligned}
d \mathbf{f}_{\| s} & =\varepsilon_{\| \mid} \mathbf{v}_{\| s} d s \\
d \mathbf{f}_{\perp s} & =\varepsilon_{\perp} \mathbf{v}_{\perp s} d s
\end{aligned}
$$


Being $\varepsilon_{\|}$and $\varepsilon_{\perp}$ the parallel and perpendicular drag coefficients. Finally, when having the force expressions integrate them over the whole swimmer length thus obtaining the total force. Though the approximation is feasible for long micrometer helices, they just have considered the helix crosssection as circular since they use the rod drag coefficients. These approximations do not take into account the effect on the swimming performances of different cross-section geometries with the same area. So, this work proposes a complete formulation of the problem in order to consider the crosssection effect on swimmer performances.

The real problem is based on the interaction of the helix structure with the fluid, fluid-structure interaction (FSI) problem. That implies Navier-Stokes $(\mathrm{N}-\mathrm{S})$ equations $\left(\Omega_{f}\right.$ fluid domain), the rotating structure dynamic $\left(\Omega_{s}\right.$ structure domain) and a boundary condition ( $\partial \Omega_{f s}$ fluid-structure boundary) that relates both equations through all time evolution $(\tau$ time interval). N-S equations describe fluid dynamics and are composed of the momentum conservation and the mass conservation equation. They are expressed as follows:

$$
\begin{gathered}
\frac{\partial \mathbf{u}}{\partial t}+\rho(\mathbf{u} . \nabla) \mathbf{u}=\nabla \cdot \Gamma+\mathbf{F} \quad \text { in } \Omega_{f} \times \tau \\
\frac{\partial \rho}{\partial t}+\nabla(\rho \cdot \mathbf{u})=0 \text { in } \Omega_{f} \times \tau \\
\Gamma=-p \mathbf{I}+\mu\left(\nabla \mathbf{u}+(\nabla \mathbf{u})^{T}\right)-\frac{2}{3} \mu(\nabla \cdot \mathbf{u})
\end{gathered}
$$

Where $\mathbf{u}$ is the fluid velocity, $p$ the pressure, $\rho$ the density, $\mu$ the dynamic viscosity, $\Gamma$ depicting the stress on an infinitesimal fluid volume ( $\nabla . \Gamma$ surface forces) and $\mathbf{F}$ the volume forces. However, when studying these equations in a dimensionless fashion, a dimensionless quantity appears called Reynolds number. This quantity gives an idea of the viscosity $f_{v i s}$ and inertial $f_{\text {in }}$ forces. Its definition is given by:

$$
R e=\frac{f_{\text {in }}}{f_{\text {vis }}}=\frac{\rho V L}{\mu}
$$

Where $V$ is the swimmer robot velocity and $L$ its characteristic length. Considering the volume force effects are negligible such it used to happen at the microscale, the momentum equation can be reduced to:

$$
R e\left(\frac{\partial \mathbf{u}^{*}}{\partial t}+\rho\left(\mathbf{u}^{*} \cdot \nabla\right) \mathbf{u}^{*}\right)=-\nabla p^{*}+\nabla^{2} \mathbf{u}^{*} \text { in } \Omega_{f} \times \tau
$$

If $R e \ll 1$, viscous effects dominate over inertial ones, the final expression can be rewritten as:

$$
\nabla p^{*}=\nabla^{2} \mathbf{u}^{*} \quad \text { in } \quad \Omega_{f} \times \tau
$$

The notation ${ }^{*}$ is for the dimensionless quantities.

Concerning the rigid robot structure, a rotation movement is along the robot helix axis. That can be translated in a prescribed mesh motion $(d \mathbf{X})$ since there is no structure deformation.

$$
d \mathbf{X}=d \mathbf{X}\left(\mathbf{r}_{\mathbf{b p}}, \omega, t\right) \quad \text { in } \quad \Omega_{m} \times \tau
$$

Where $\mathbf{r}_{\mathbf{b p}}$ is the helix axis, $\omega$ is the angular frequency and $t$ is time and $\Omega_{m} \subset \Omega_{s}$. The boundary condition in $\partial \Omega_{f s}$ that stands for the coupling between $\mathrm{N}-\mathrm{S}$ equations and the structure movement is given by:

$$
\begin{gathered}
\mathbf{u}=\mathbf{u}_{s} \text { in } \partial \Omega_{f s} \times \tau \\
\mathbf{u}_{s}=\frac{\partial \mathbf{X}}{\partial t} \quad \text { in } \quad \partial \Omega_{f s} \times \tau
\end{gathered}
$$

Where $\mathbf{u}_{s}$ is the solid structure velocity in one point situated on its surface. Another boundary condition in the limits of the container $\left(\partial \Omega_{f}\right)$ is:

$$
\mathbf{u}=\mathbf{0} \quad \text { in } \quad \partial \Omega_{f} \times \tau
$$

Null fluid speed in the fluid-container boundary. Finally, force components are computed by integrating the total stress $(\Gamma)$ over all the robot's surface.

$$
F_{i}=\int_{\partial \Omega_{s}} \Gamma \mathbf{n}_{f} \cdot \mathbf{e}_{i} d s \text { in } \partial \Omega_{f s} \times \tau
$$

Being $\mathrm{i}=\mathrm{x}, \mathrm{y}$, and $\mathrm{z}$ for all different components, $\mathbf{n}_{f}$ the normal vector to the surface of each finite element on the robot surface, $d s$ the surface element, and finally, $\mathbf{e}_{i}$ representing the unit vectors for $\mathrm{x}, \mathrm{y}$, and $\mathrm{z}$ of the global frame.

\section{EXPERIMENTAL AND SIMULATION RESULTS}

This current study deals with helical swimmers with different cross-sections in order to see their influence on the propulsion speed (via experiments) and the computed propulsion force (via FEM simulations in COMSOL). The simulations have considered a fixed helical structure rotating about the helix principal axis, then the stress generated along all its surface is computed in the stationary regime and integrate it to have the total force in all directions. Because the simulations only consider the rotational motion that is imposed on the robot, there is not exists cut-off frequency. So, cut-off frequencies are imposed based on the experimental results.

\section{A. Different cross-section geometries study}

The first experiment considers three helical swimmers with practically the same weight but different cross-section geometries, namely an equilateral triangle, a square, and a circle. In order to achieve the same weight for all robots, we have considered the same value of cross-section area before their sweeping over the helix spine. It means if we consider a circle with radius $r$, then a square with side $l=1.77 r$ and an equilateral triangle with side $L=2.69 r$ will have the same area. Moreover, the surface area that interacts with the surrounding fluid for each robot after sweeping will be proportional to their perimeter (table I). In our case the circular cross-section radius is $0.4 \mathrm{~mm}$ while the considered helix parameterization in its general form is:

$$
\begin{gathered}
x=a \cos (t) \\
y=a \sin (t) \\
z=c t
\end{gathered}
$$

Where $a, c$ and $t \in \mathbb{R}$. In the conceived helices, these values are $a=0.6 \mathrm{~mm}, c=4 \mathrm{~mm}$ and $t \epsilon[0,2 \pi n]$ with $n=2$-turns. 
(a)

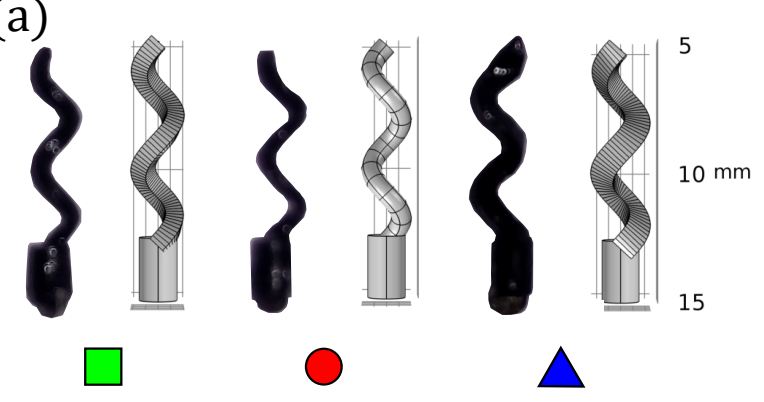

(b)



(c)
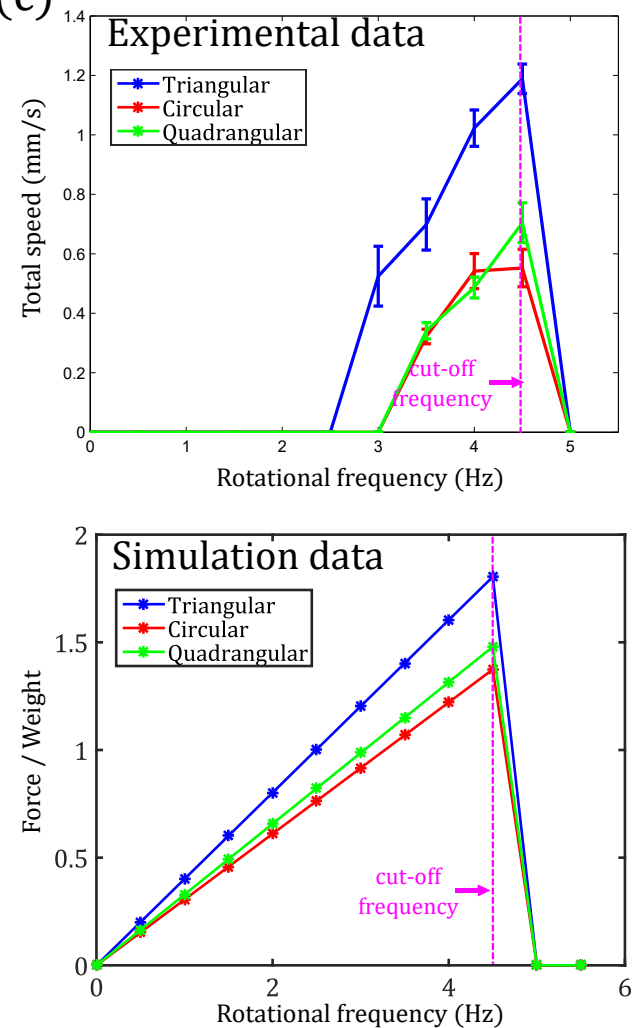

Fig. 2. First experiment considering 3 helical swimmers with practically the same weight and different cross-section geometries. (a) Conceived robots and their respective CAD designs for different cross-sections, there exists some differences because of the 3D impression resolution. (b) A bar graph representing the weight and volume of each robot. (c) Experimental data and simulation results, the former depicts the total speed vs. rotation frequency while the latter shows Force/Weight vs. the rotation frequency. For each helical robot, the experiments were performed three times for each frequency, and the error bar is the standard deviation of those trials. Besides, the force was computed by integration of the stress generated on the robot surface by its rotation within the viscous medium and then divided by the robot's weights.

TABLE I

DIFFERENT CROSS-SECTION PERIMETERS

\begin{tabular}{ccc}
\hline Circle & $\begin{array}{c}\text { Perimeter } \\
\text { Triangle }\end{array}$ & Square \\
\hline $6.28 \mathrm{r}$ & $8.08 \mathrm{r}$ & $7.08 \mathrm{r}$ \\
\hline
\end{tabular}

Fig. 2.a depicts the three printed swimmer and their respective CAD representations. The weight and its volume were measured using a precision balance and the CAD, respectively. These results are depicted in Fig. 2.b. There is a slight difference of $1 \mathrm{mg}$ because of different factors such as the voxel precision of the printing machine and some small modifications in the CAD because of wrong boundary errors when printing due to the printer software. However, despite these small differences, experimental and simulation results have shown better performances in terms of speed and ratio Force/Weight for the helical robot with a triangular cross-section (cf. Fig.2.c). Knowing that forces on the robots depend upon the interaction of the fluid with their surfaces. There exists a correlation between the surface area of the helical structures and the forces acting on them. Having a greater helical-shaped surface, the forces on the surface will increase according to the force expression in 14. So, helical swimmer with an equilateral cross-section, which generates a greater surface than other cross-section geometries, is exposed to interact more with the fluid achieving a higher value of propulsion force, and so, corroborating the obtained results.

Fig. 3.a depicts the fluid speed time-evolution around the rotating helical structure with a triangular cross-section. Furthermore, in order to see the velocity field, the cross and axial planes views are represented in Fig. 3.c. Finally, to know how the force evolves, the graph 3.b shows the forces in $\mathrm{x}$, $\mathrm{y}$, and z-direction being this latter the propulsion force direction.

\section{B. New conical helix analysis of the number of turns}

This second experiment consists of the analysis of a new potential prospect of the helical swimmer for delivery tasks due to its growing radial pitch that allows the robot to carry bigger objects inside its flagellum than the common helix design. The conical helix equation is given in general by next parameterization:

$$
\begin{gathered}
x=(\beta+\alpha t) \cos (t) \\
y=(\beta+\alpha t) \sin (t) \\
z=c t
\end{gathered}
$$




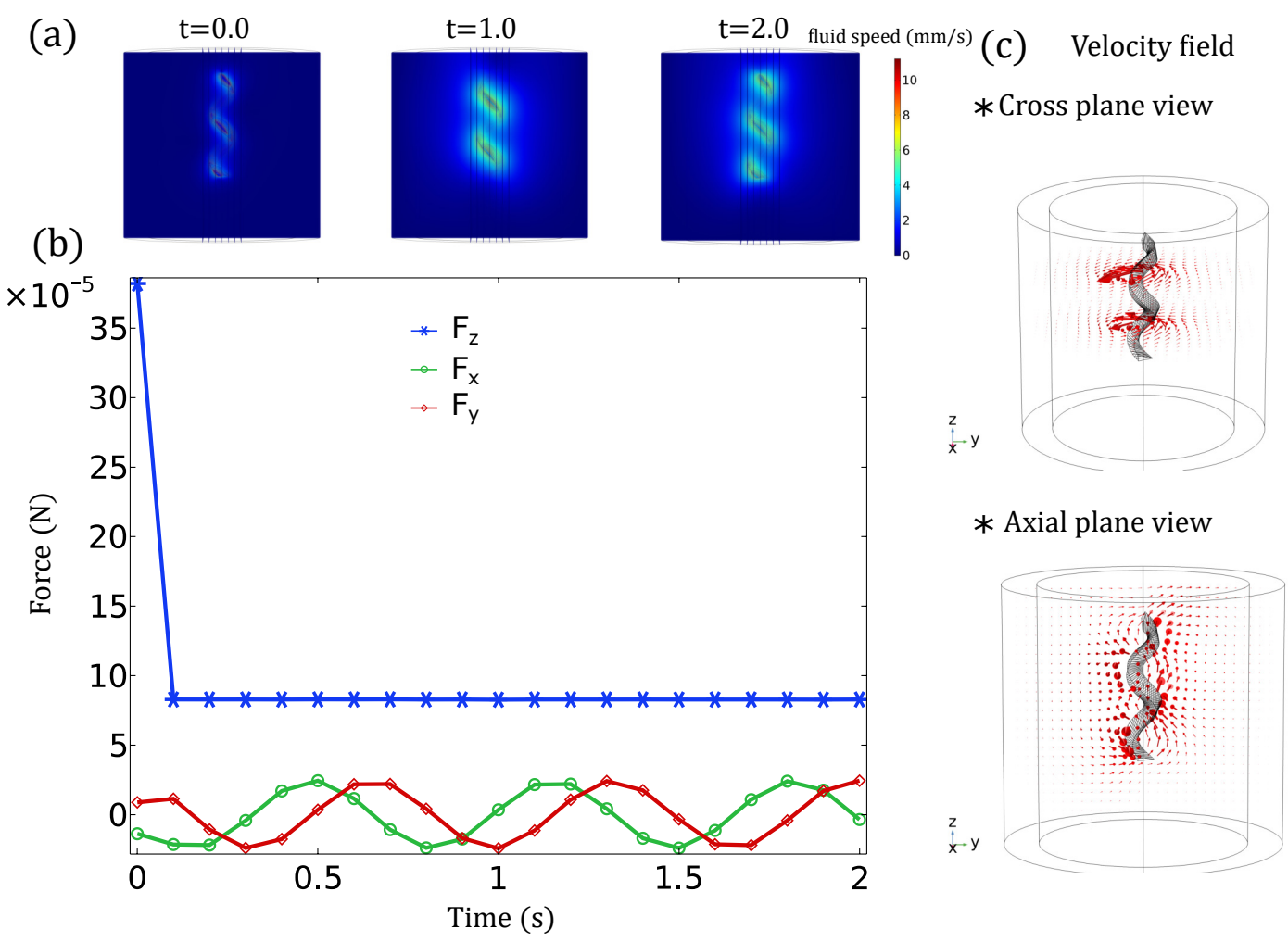

Fig. 3. Time evolution analysis for a helical structure rotating at 1.5-turns/s. (a) Color representation of the fluid speed around the rotational motion of the robot structure with a triangular section for different times. (b) Force evolution through the time for x, y, and z-axis. (c) Velocity field view in two cross-sectional planes (top part) and one axial cut (bottom part).

Where $\alpha, \beta$ and $t \in \mathbb{R}$. In our particular case $\beta=0$, $\alpha=1 / 2 \pi, c=4 / 2 \pi$ and $t=[0,2 n \pi]$ where $\mathrm{n}$ is the number of turns and all $\mathrm{x}, \mathrm{y}$, and $\mathrm{z}$ components were measured in millimeters. The tested number of turns are 1, 1.5, 2.5, 3.5 (cf. Fig4.a). The experiments are carried out considering an inclination angle defined between the helix axis and the $\mathrm{z}$ axis of the global frame of $20^{\circ}$. The total propulsion speed is measured and depicted in figure 4.c. The cut-off frequency is different for each helix because it only depends on the magnetic force and the helix inertia of each robot. On the other hand, simulation results only compute the force generated due to the fluid interaction regardless the inclination angle and the apparent weight that we suppose negligible since the fluid density value $\left(1260 \mathrm{Kg} / \mathrm{m}^{3}\right)$ is closed to the printing material density value $\left(1100 \mathrm{Kg} / \mathrm{m}^{3}\right)$. Figure $4 . \mathrm{b}$ shows how the total force evolves as function of the number of turns and the rotation frequency without considering the experimental cutoff frequencies. When considering these cut-off frequencies, the fluidic forces acting on the robot surface are computed and then divided by the weight of each robot respectively, thus obtaining the normalized graph 4.d. These last results agree with the experimental data showing that the best performances are achieved by the 1-turn and 1.5-turns corroborating as well the previous results achieved in [1], [3], [14].

\section{CONCLUSIONS AND CONTRIBUTION}

This work has investigated the effect of the cross-section geometry on swimming performances of magnetic helical robots with the same mass. Experiments and simulations show that robots with equilateral triangle cross-sections generate better propulsion (in terms of propulsion speed and force) than circular ones with the same area. The speed reached experimentally for these improved robots is about $1.2 \mathrm{~mm} / \mathrm{s}$, which is practically two times the speed reached for helices with circular and quadrangular cross-sections. Simulations show that fluidic force on the robot surface experience the same growth trend as the total speed curve for these robots (the largest fluidic force and total speed values for the helical robot with a triangular cross-section). Furthermore, another study concerning the conical spiral is developed. The effect of the number of turns has been studied for this new kind of helix showing that 1.0 and 1.5-turns have better performances than the other helices. The speed achieved experimentally for these last improved robots is about $2.0 \mathrm{~mm} / \mathrm{s}$ and the simulation results show a correspondence between the fluidic normalized force and the velocities in a linear fashion. Though this one-turn flagellum has reached the greatest speed value over all experiments, the multi-turn ones could serve in future applications for delivery tasks thanks to its growing radial pitch. They also could experience better propulsion if the magnetic force were incremented. Finally, both studies for the 
(a)

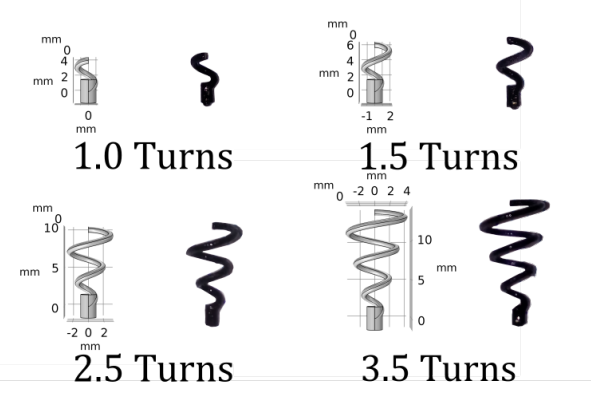



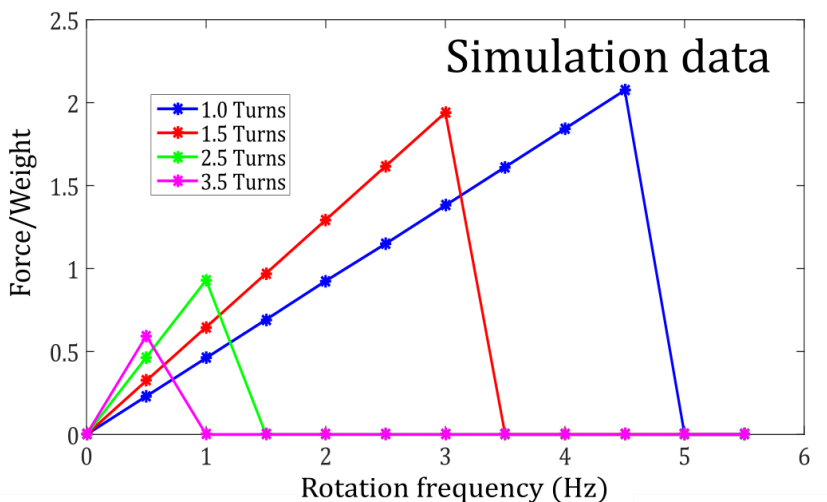

Fig. 4. Second experiment considering different number of turns for a conical helix, (a) depicts the CAD representation and the respective conical helical robot next to it for 1.0, 1.5, 2.5, and 3.5-turns, (b) stands for the total force exerted on the swimmer surface as function of the number of turns and the rotation frequency without considering the cut-off frequencies. (c) shows the rotation frequency vs the total force for the conical helices obtained experimentally. As in the previous experiments, for each conical helix, the experiments were performed three times for each frequency, and the error bar is the standard deviation of those trials. (d) represents the rotation frequency vs Force/Weight for each robot by simulations, for this curve, we have taken the cut-off frequencies from the experiments.

cross-section analysis and the number of turns for the conical helix have been conceived for the first time in literature, being this our contribution.

\section{REFERENCES}

[1] Ambarish Ghosh and Peer Fischer. Controlled propulsion of artificial magnetic nanostructured propellers. Nano letters, 9(6):2243-2245, 2009.

[2] Eric E Keaveny, Shawn W Walker, and Michael J Shelley. Optimization of chiral structures for microscale propulsion. Nano letters, 13(2):531537, 2013.

[3] Nguyen Hao Le, James Ritchie, and Jining Sun. Shape optimisation of biocompatible/degradable helical micro/nano-structures for drug delivery. Nanoscience and Nanotechnology Letters, 9(4):496-501, 2017.

[4] Veronika Magdanz, Mariana Medina-Sánchez, Lukas Schwarz, Haifeng $\mathrm{Xu}$, Jens Elgeti, and Oliver G Schmidt. Spermatozoa as functional components of robotic microswimmers. Advanced Materials, 29(24):1606301, 2017.

[5] Arthur W Mahoney, John C Sarrazin, Eberhard Bamberg, and Jake J Abbott. Velocity control with gravity compensation for magnetic helical microswimmers. Advanced Robotics, 25(8):1007-1028, 2011.

[6] Bradley J Nelson, Ioannis K Kaliakatsos, and Jake J Abbott. Microrobots for minimally invasive medicine. Annual review of biomedical engineering, 12:55-85, 2010.

[7] Ali Oulmas, Nicolas Andreff, and Stéphane Régnier. Closed-loop 3d path following of scaled-up helical microswimmers. In Robotics and Automation (ICRA), 2016 IEEE International Conference on, pages 1725-1730. IEEE, 2016.

[8] Salvador Pané, Josep Puigmartí-Luis, Christos Bergeles, Xiang-Zhong Chen, Eva Pellicer, Jordi Sort, Vanda Počepcová, Antoine Ferreira, and Bradley J Nelson. Imaging technologies for biomedical micro-and nanoswimmers. Advanced Materials Technologies, page 1800575.
[9] Edward M. Purcell. Life at low Reynolds number. American Journal of Physics, 45(1):3-11, January 1977.

[10] Famin Qiu and Bradley J Nelson. Magnetic helical micro-and nanorobots: Toward their biomedical applications. Engineering, 1(1):021-026, 2015.

[11] David Russell and Are von der Lippe. Cluster headache: heart rate and blood pressure changes during spontaneous attacks. Cephalalgia, 2(2):61-70, 1982

[12] Fatma Zeynep Temel and Serhat Yesilyurt. Simulation-based analysis of micro-robots swimming at the center and near the wall of circular mini-channels. Microfluidics and Nanofluidics, 14(1-2):287-298, 2013.

[13] Soichiro Tottori, Li Zhang, Famin Qiu, Krzysztof K Krawczyk, Alfredo Franco-Obregón, and Bradley J Nelson. Magnetic helical micromachines: fabrication, controlled swimming, and cargo transport. Advanced materials, 24(6):811-816, 2012.

[14] D Walker, M Kubler, KI Morozov, P Fischer, and AM Leshansky. Optimal length of low reynolds number nanopropellers. Nano letters, 15(7):4412-4416, 2015.

[15] Tiantian Xu, Gilgueng Hwang, Nicolas Andreff, and Stéphane Régnier. Modeling and swimming property characterizations of scaled-up helical microswimmers. IEEE/ASME Transactions on Mechatronics, 19(3):1069-1079, 2014.

[16] Tiantian Xu, Gilgueng Hwang, Nicolas Andreff, and Stéphane Régnier. Influence of geometry on swimming performance of helical swimmers using doe. Journal of Micro-Bio Robotics, 11(1-4):57-66, 2016.

[17] Xiaohui Yan, Qi Zhou, Jiangfan Yu, Tiantian Xu, Yan Deng, Tao Tang, Qian Feng, Liming Bian, Yan Zhang, Antoine Ferreira, et al. Magnetite nanostructured porous hollow helical microswimmers for targeted delivery. Advanced Functional Materials, 25(33):5333-5342, 2015. 